\title{
Facial Pityriasis Alba, Polymorphous Light Eruption, and Vitiligo in Children: A Dermoscopic Distinction
}

\author{
Balachandra Suryakant Ankad (iD ${ }^{1,{ }^{*}}$, Sankappanavara V Smitha ${ }^{1}$, Enzo Errichetti (iD) ${ }^{2}$ and Manjula \\ Rangappa ${ }^{3}$ \\ ${ }^{1}$ Department of Dermatology, S N Medical College, Bagalkot, 587102, Karnataka, India \\ ${ }^{2}$ Institute of Dermatology, University Hospital Santa Maria della Misericordia, Udine, Italy \\ ${ }^{3}$ Department of Community Medicine, S N Medical College, Bagalkot, India \\ "Corresponding author: Department of Dermatology, S N Medical College, Bagalkot, 587102, Karnataka, India. Email: drbsankad@gmail.com
}

Received 2021 December 12; Revised 2022 January 16; Accepted 2022 January 16.

\begin{abstract}
Background: Hypopigmentary skin disorders are the most worrisome complaints from Indian and Indian subcontinent people. Pityriasis alba (PA), polymorphic light eruption (PLE), and vitiligo are clinically look-alike conditions commonly seen in children. Objectives: We attempted to characterize the dermoscopic features of PA and PLE and differentiate them from vitiligo in the facial region in skin of color.

Methods: Dermoscopic evaluation was done using a handheld dermoscope at 10X magnification on facial lesions of a total of 60 patients with PA, PLE, and vitiligo. Dermoscopic features of all three conditions were compared and correlated with histological features.

Results: Out of 60 patients, 30 (50\%), 20 (33.33\%), and 10 (16.66\%) patients were diagnosed with PA, PLE, and vitiligo clinically and histologically, respectively. Dermoscopy of PA showed white structureless areas (100\%), ill-defined margins (86\%), faint brownish pigmentation $(70 \%)$, and fine scales $(70 \%)$ (P value $=0.001)$. Besides, 70\% of PLE cases showed white structureless areas and ill-defined margins, and $10 \%$ showed faint brownish pigmentation (P value $=0.0001)$. Coarse scales and clustered dots were the most common findings (75\%) in PLE lesions, followed by light brown background (60\%), crusts (40\%), and yellow clods (30\%) (Pvalue $=0.0001)$. Also, $40 \%$ and $35 \%$ of the PLE cases showed white and brown course scales, respectively (P value $=0.0001$ ). Moreover, $13.33 \%$ of PA cases showed coarse white scales, and 3.33\% showed a light brown background. All vitiligo cases showed a white glow appearance (100\%), followed by perifollicular pigmentation, leukotrichia, and koebnerization shown by $40 \%$ of the cases (P value $=0.0001)$. Finally, $20 \%$ of the cases showed perilesional pigmentation and satellite lesions.

Conclusions: PA lesions are dermoscopically characterized by ill-defined white areas with fine branny scales, whereas PLE shows coarse brown scales, ring scales, crusts, and yellow clods along with the above findings. Vitiligo lesions are devoid of scales with various pigment network abnormalities, perilesional and perifollicular pigmentation, and leukotrichia.
\end{abstract}

Keywords: Dermoscopy, Pityriasis Alba, Polymorphous Light Eruption, Vitiligo

\section{Background}

Hypopigmentary skin disorders are the most worrisome complaints given by patients with skin of color. They are of great concern and anxiety when they appear in the facial region because of religious and cultural customs, disturbing the peace of mind. The most common hypopigmented or depigmented lesions affecting children's faces include pityriasis alba (PA), polymorphic light eruption (PLE), and vitiligo. Pityriasis alba has no definitive treatment but resolves spontaneously over months to years (1). Polymorphic light eruption is commonly seen in tropical countries during the summer months. It presents as asymptomatic hypopigmented patches on sun-exposed areas, mainly the face (2). Vitiligo is an autoimmune disease occurring on any site of the body but causes immense cosmetic disfigurement if it occurs on the face (3). Although the diagnosis is straightforward based on the clinical features, few atypical manifestations pose a diagnostic challenge. This mandates the histopathological study for which willingness for skin biopsy is a drawback, especially in children. Since treatment and prognosis are different in PA, PLE, and vitiligo, it is of paramount importance to arrive at an accurate diagnosis.

Dermoscopy is a recent addition to the diagnostic armamentarium in dermatology. It acts as an adjunctive 
method in the accurate diagnosis of many inflammatory dermatoses (4). There are many evidence-based descriptions of dermoscopic patterns in PA and vitiligo. However, dermoscopy of PLE is limited to case reports (5). Here, the authors attempted to evaluate the dermoscopic features of these conditions and show how best dermoscopy helps in their differentiation.

\section{Objectives}

We aimed to characterize the dermoscopic features of PA and PLE and differentiate them from vitiligo in the facial region in skin color.

\section{Methods}

This was a cross-sectional controlled study conducted in a tertiary hospital in Southern India. The study was conducted from August 2020 to March 2021 for eight months. Institutional ethical committee clearance was taken. Written informed consent was obtained from patients and concerned guardians. All patients aged $>1$ year and $<20$ years with suspected facial lesions of PA, PLE, and vitiligo were enrolled in the study. The diagnosis was made by an experienced dermatologist (BSA) based on the clinical criteria for each condition (1-3). Patients who had received treatment in the previous month and had lesions associated with infection were excluded from the study. Demographic particulars, including age, sex, disease duration, and associated illness, were noted down. Detailed clinical examination was done, and required hematological investigations were carried out.

Clinical images were captured with a digital camera, and dermoscopy was done with a handheld (Illuco 1100 IDS and FotoFinder Handyscope) dermoscope with 10X magnification attached to a smartphone in the polarized mode under aseptic precautions. The dermoscopic assessment was carried out by experienced dermoscopists (BSA and EE). The dermoscopic variables were selected according to the recent validation by International Dermoscopy Society criteria in skin of color (4).

\subsection{Statistics}

Dermoscopic features were tabulated and fed into SPSS statistics v20.0 for Windows (SPSS Inc., Chicago, USA). Continuous variables such as age and duration are described as means \pm standard deviations. Discrete variables are shown as percentages. The P value $<0.05$ was considered statistically significant.

\section{Results}

A total of 60 patients were recruited in the study, including 33 (55\%) males and 27 (45\%) females. All of them had Fitzpatrick IV and V skin types. The majority of the patients (50\%) were in the 6 to 10-years-old age group. Out of 60 patients, 30 (50\%), 20 (33.33\%), 10 (16.66\%) patients were diagnosed clinically with PA, PLE, and vitiligo, respectively.

Dermoscopy of PA showed white structureless areas in all (100\%) patients as the most common feature, and satellite lesion was noted in a single (3.33\%) patient (Figure 1). Clustered red dots and white structureless areas were noted in 15 (75\%) and 14 (70\%) PLE patients, respectively (Figure 2). All the 10 (100\%) vitiligo lesions demonstrated white-structureless areas (Figures 3 - 5). Three (30\%) vitiligo lesions had a faint pigment network (Figures $4 \mathrm{~B}$ and $5 \mathrm{~A}$ ).

Scales were both fine and coarse in nature. The former was exclusively seen in four (13.33\%) PA cases, and the latter was found in 15 (75\%) PLE cases, with a P value of 0.0001 . None of the vitiligo lesions showed fine scales. Perifollicular pigmentation (Figures 3B and 5B), leukotrichia (Figures $3 \mathrm{~B}$ and $4 \mathrm{~B})$, and Koebnerisation were noted only in vitiligo lesions $(\mathrm{P}=0.0001)$. Likewise, a 'yellow clod' sign and clustered red dots (Figure $2 \mathrm{~B}$ and $\mathrm{F}$ ) were noted only in PLE lesions with a $\mathrm{P}$ value of 0.0001 . Perilesional pigmentation and satellite lesions in PA and vitiligo were seen in variable frequencies, which were not statistically significant. Different dermoscopic features in PA, PLE, and vitiligo are depicted in Table 1.

\section{Discussion}

Dermoscopy, also known as epiluminescence microscopy, is a non-invasive, on-table diagnostic tool that involves a handheld device with built-in illumination and magnification system (1). It is used to visualize skin at different levels with the help of polarized light. Dermoscopy initially aided in diagnosing pigmented lesions and melanoma, but now it has much importance in inflammatory and infective dermatoses. It not only assists in diagnosis but also helps in treatment evaluation and disease prognosis (6).

Usually, PA is an incidental finding characterized by scaly macules with well-defined borders on the face (7). It is usually a clinical diagnosis; however, histopathology is helpful in doubtful cases. Histology shows hyperkeratosis, parakeratosis, and acanthosis. Spongiosis can be seen occasionally. Melanocytes and melanosomes are present in decreased amounts in the basal layer of the epidermis (8). As mentioned above, PA demonstrates white structureless areas, ill-defined borders, and a faint pigment network 

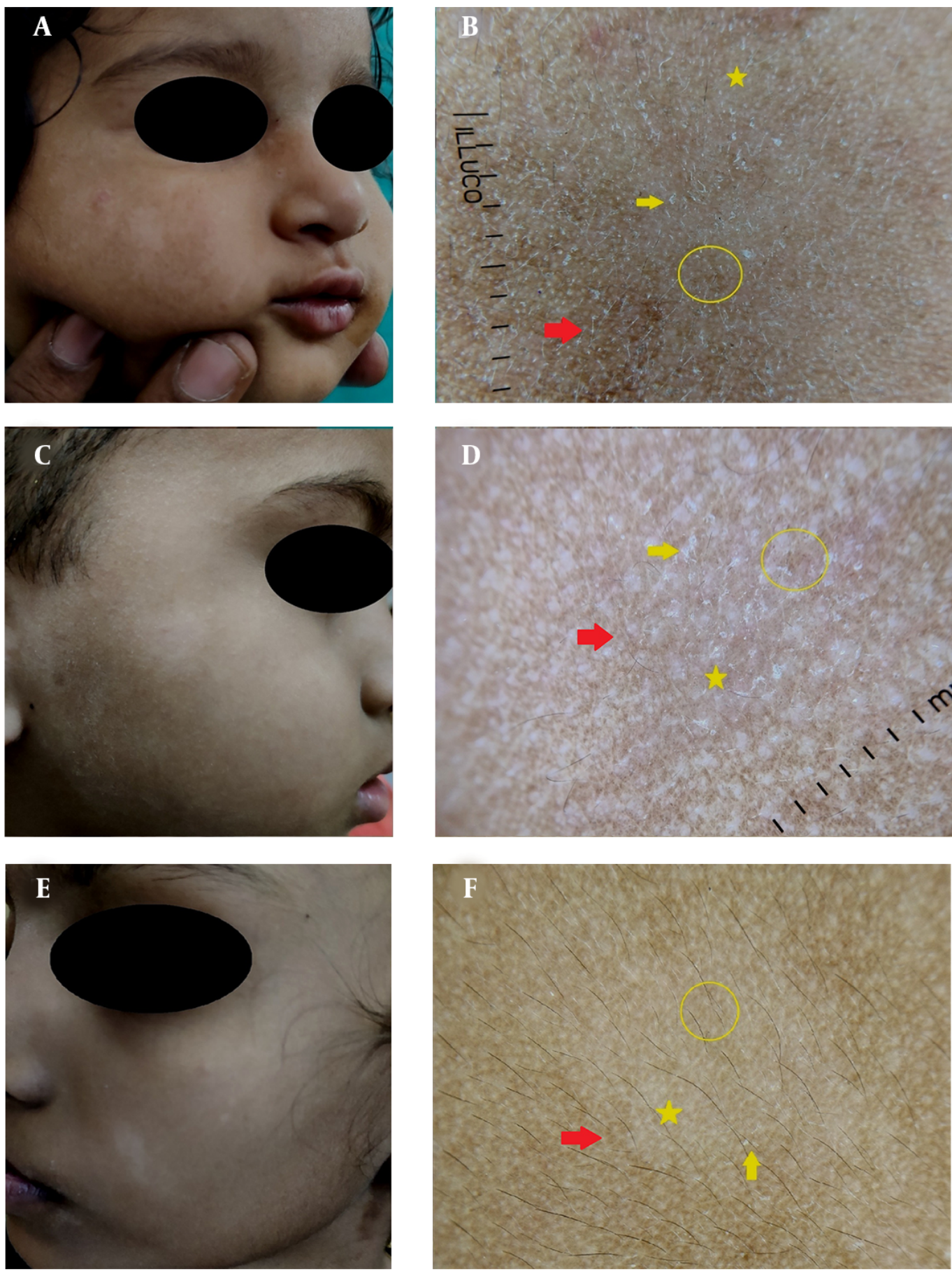

Figure 1. A, C, and E, Clinical images of pityriasis alba showing hypopigmented patches on the face; B, D, and F, Corresponding dermoscopy showing a faint pigment network (yellow circles), white areas (yellow stars), and fine white scales (yellow arrows). Ill-defined borders (red arrows) and brown backgrounds are well appreciated.

with fine white scales in dermoscopy (9). White structureless areas correspond to acanthosis and decreased melanin in the epidermis, and fine scales are due to hyperkeratosis and parakeratosis. The faintness of the pigment network is attributed to acanthosis with lessened melanin in the affected area. 

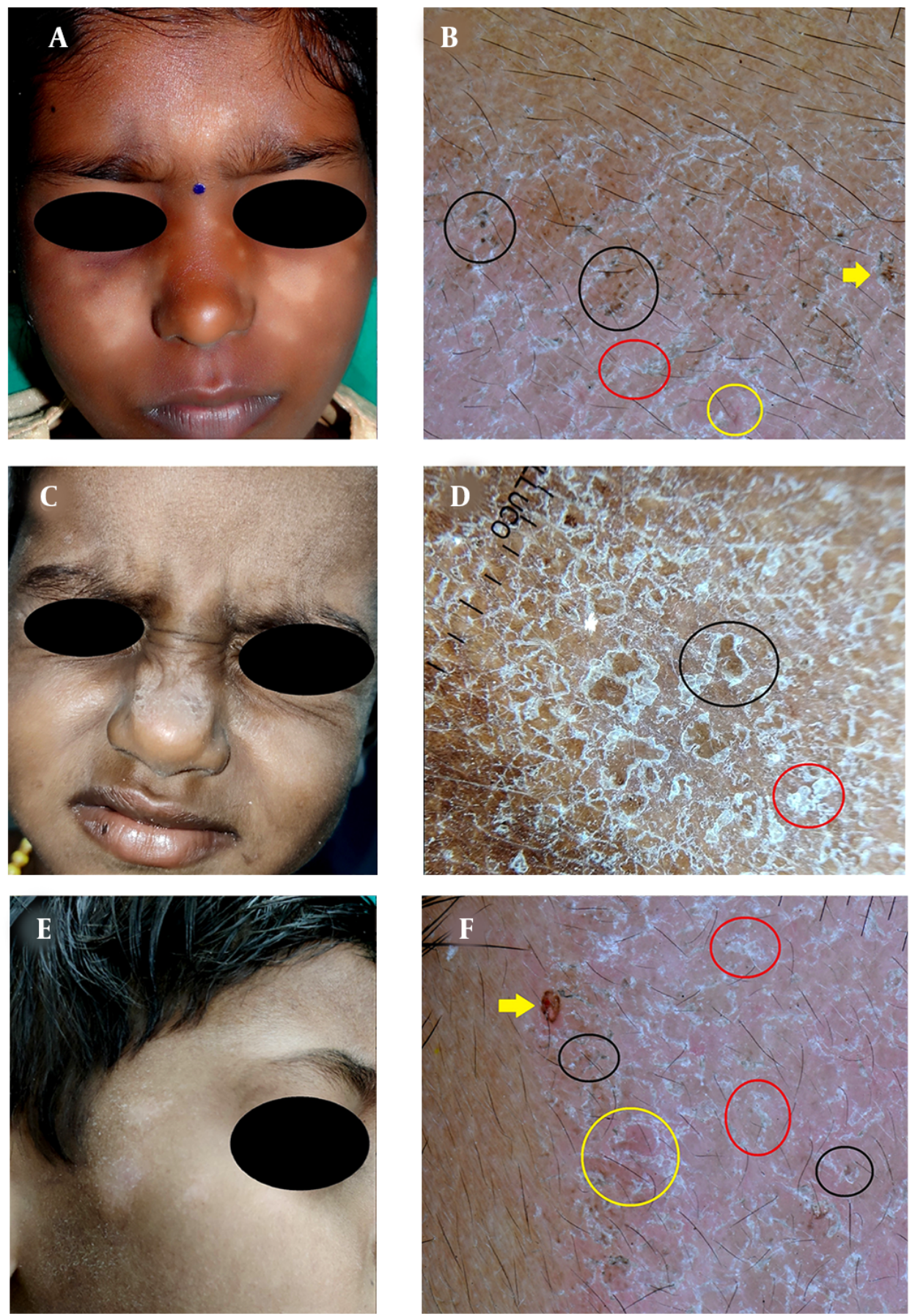

Figure 2. A, C, and E, Clinical images of polymorphous light eruption showing hypopigmented patches on the face; B, D, and F, Corresponding dermoscopy showing yellow clod signs (serocrusts) (yellow arrows), clustered red dots (yellow circles), and coarse brown scales (black circles). Note the coarse white scales (red circles) and the light brown background.

Besides, PLE is the most common, idiopathic condition due to recurrent and delayed reaction to sunlight (2). It is characterized by pruritic, erythematous papules, vesi- cles, or plaques (10). The histopathology of PLE is characterized by hyperkeratosis, acanthosis and spongiosis with or without liquefactive degeneration in the epidermis. Der- 

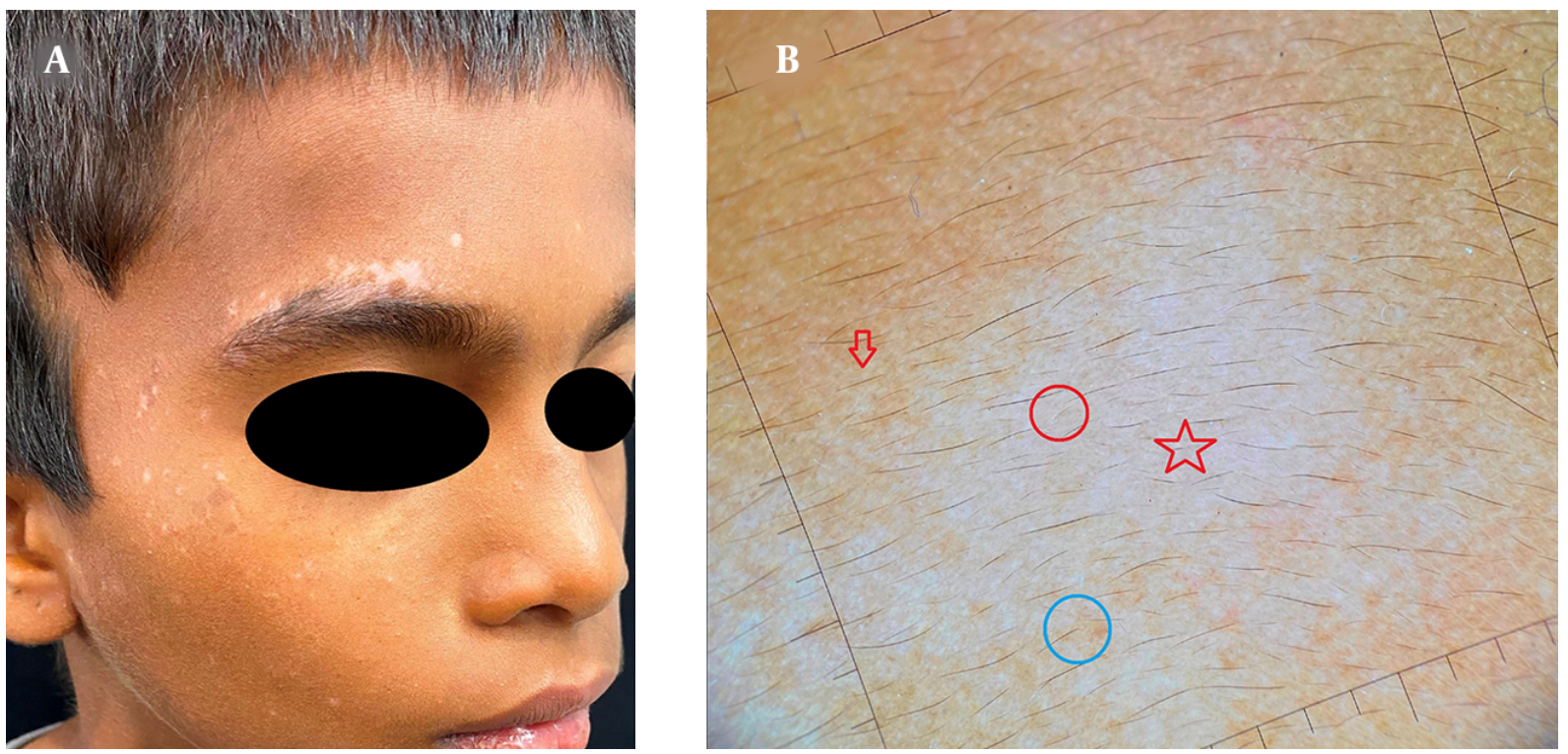

Figure 3. A, Clinical image of vitiligo showing depigmented patch on the face; B, Dermoscopy showing white glow (red star), ill-defined border (red arrow), and perifollicular pigmentation (blue circle). Leucotrichia (red circle) is well appreciated.
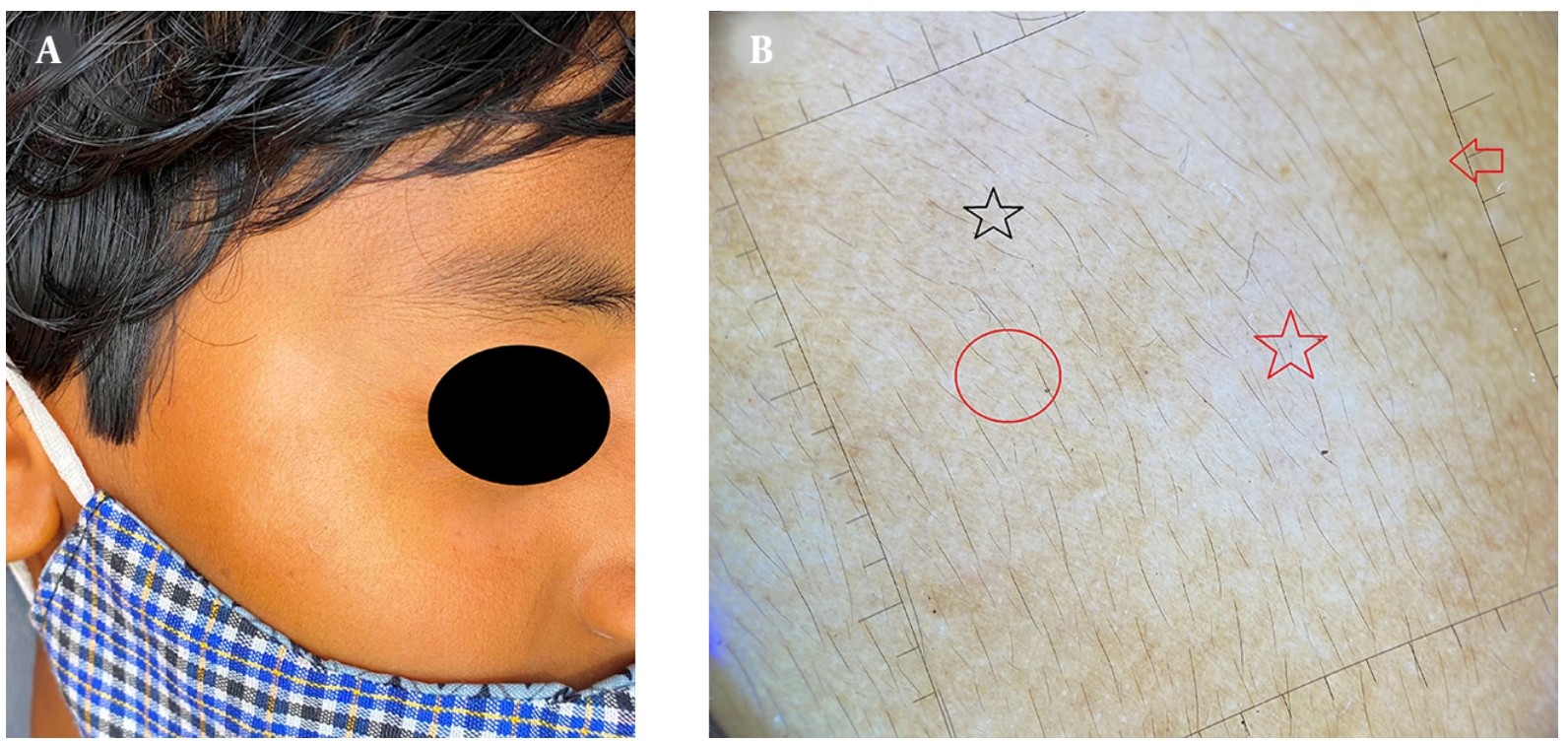

Figure 4. A, Clinical image of vitiligo showing depigmented patch on the face; B, Dermoscopy showing a faint pigment network (black star), white glow (red star), ill-defined border (red arrow), and leukotrichia (red circle).

mal changes in the upper and mid dermis include the presence of dense perivascular lymphocytic infiltrate composed primarily of T lymphocytes (2). Dermoscopy of PLE is described in the lesions affecting the forearm. The authors noted white areas, clustered red dots, and ring scales (5). Nevertheless, this report describes the dermoscopic features in PLE lesions affecting the forearm.

We found well to ill-defined white structureless areas with coarse brown and white scales in a diffuse distribution in the lesions confined to the face. Yellow clod signs or serocrusts and clustered red dots were the other features. The pigment network was light brown in the background. 

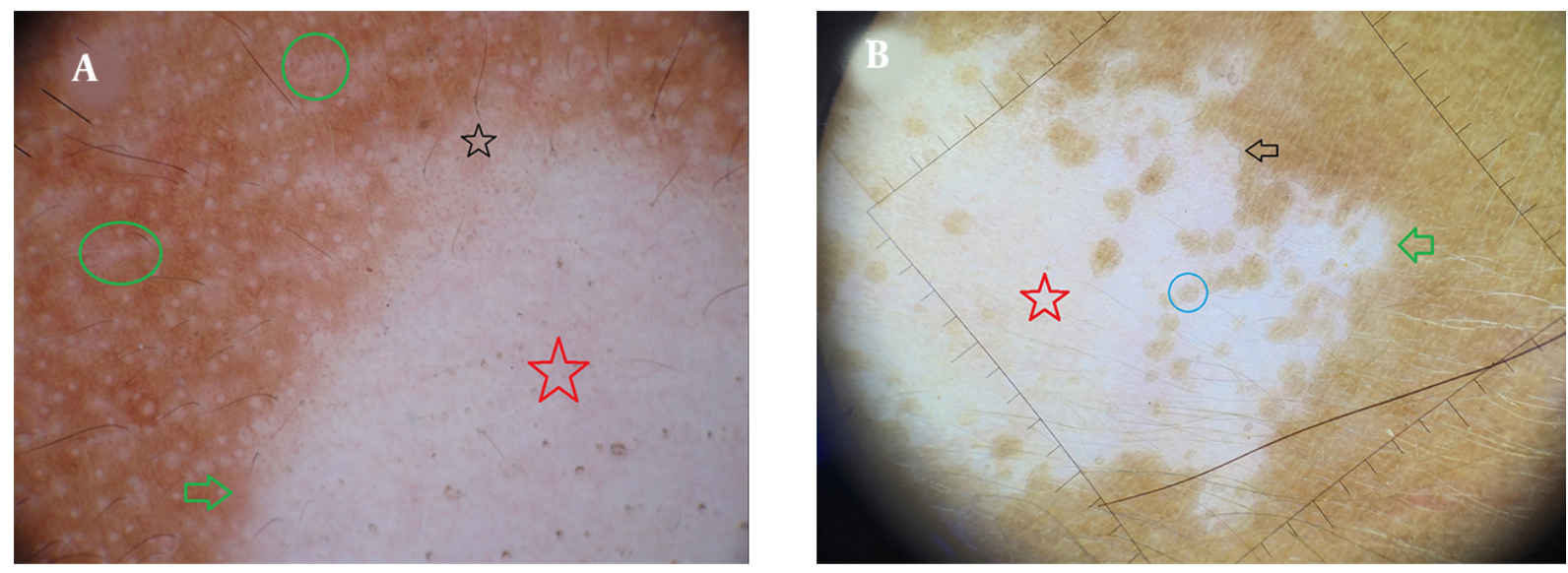

Figure 5. A and B, Dermoscopy of vitiligo showing white glow(red stars), faint pigment network(black star), ill-defined border (green arrows), and perifollicular pigmentation (blue circle). Note the satellite lesions (green circles) and perilesional pigmentation (black arrow).

Table 1. Dermoscopic Characteristics of Facial Pityriasis Alba, Polymorphous Light Eruption, and Vitiligo ${ }^{\text {a }}$

\begin{tabular}{|c|c|c|c|c|c|c|}
\hline Dermoscopic Characteristics & P. Alba (30) & PLE (20) & Vitiligo (10) & Total $(60)$ & Chi-Square Value & P-Value \\
\hline White structureless areas & $30(100)$ & $14(70)$ & $10(100)$ & 54 & 13.33 & 0.001 \\
\hline Ill-defined margins & $26(86)$ & $14(70)$ & $2(20)$ & 42 & 15.87 & 0.0001 \\
\hline Faint brownish pigmentation & $21(70)$ & $2(10)$ & $3(30)$ & 25 & 18.46 & 0.001 \\
\hline Fine scales & $21(70)$ & 0 & 0 & 21 & & 0.0001 \\
\hline \multicolumn{7}{|l|}{ Coarse scales } \\
\hline White & $4(13.33)$ & $8(40)$ & 0 & 12 & 29.10 & 0.0001 \\
\hline Brown & 0 & $7(35)$ & 0 & 7 & & 0.0001 \\
\hline Light brown background & $1(3.33)$ & $12(60)$ & 0 & 13 & 26.02 & 0.0001 \\
\hline Clustered red dots & 0 & $15(75)$ & 0 & 15 & & 0.0001 \\
\hline Yellow-clod sign & 0 & $8(40)$ & 0 & 8 & 18.46 & 0.0001 \\
\hline White glow & 0 & 0 & $10(100)$ & 10 & & 0.0001 \\
\hline Perifollicular pigmentation & 0 & 0 & $4(40)$ & 4 & & 0.0001 \\
\hline Perilesional pigmentation & $1(3.33)$ & 0 & $2(20)$ & 3 & 5.96 & 0.051 \\
\hline Leukotrichia & 0 & 0 & $4(40)$ & 4 & & 0.0001 \\
\hline Satellite lesions & $1(3.33)$ & 0 & $2(20)$ & 3 & 5.965 & 0.051 \\
\hline Koebnerisation & 0 & 0 & $4(40)$ & 4 & & 0.0001 \\
\hline
\end{tabular}

${ }^{a}$ Values are expressed as No. (\%).

Notably, we did not find ring scales; instead, scales with everted edges were noted. Probably the different site of involvement is attributed to this disparity. It should be noted that scales were coarse and fine compared to PA, wherein they were fine in morphology. Interestingly, few scales had a brown hue that is probably indicative of dried serum produced due to scratching. Dermoscopic-histopathological correlation is explained as scales, and the white structureless area is due to hyperkeratosis and acanthosis. Yellow clod sign corresponds to serous fluid and spongiosis, and red dots are attributed to dilated capillaries in the dermis. The brownish background is suggestive of melanin in the epidermis.

Vitiligo is a common, acquired, autoimmune depigmentary disorder. It clinically manifests as circumscribed hypochromic or achromic macules often associated with leukotrichia. Histology shows the absence of melanin granules in vitiliginous areas, and histochemical studies 
show a gross lack of DOPA-positive melanocytes in the basal layer (11).

Dermoscopy of vitiligo shows different patterns based on disease activity. Evolving lesions show white structureless areas, reduced or absent pigmentary network, reverse pigment network; and perifollicular and perilesional hyperpigmentation, and ill-defined margins (12). New findings such as starburst appearance, comet-tail (microKoebnerization), and tapioca sago grain appearance are reported in progressive lesions (13). These features were not noted in either PA or PLE, thus affirming that these dermoscopic patterns are specific to vitiligo $(P=0.0001)$. Leukotrichia, which may not be evident clinically, was visualized better in dermoscopy. This finding is also found only in vitiligo $(\mathrm{P}=0.0001)$.

Interestingly, satellite lesions were noted in both PA and vitiligo. It is noteworthy that satellite lesions speak of the spreading nature of both vitiligo and PA. Observation of such lesions should prompt the treating clinician to modulate treatment protocol accordingly. A white structureless area without a pigment network is called 'white glow,' indicating the total absence of melanin in the basal layer. This peculiar pattern is not found in PA, PLE, and even other hypopigmented or depigmented lesions, except idiopathic guttate hypomelanosis (14). Pigmentation in the form of residual or faint pigment network is noted either in evolving vitiligo lesions (12). In this study, a few vitiligo lesions $(n=3)$ showed it. Thus, a faint pigment network under dermoscopy implies early lesions, and treatment should be offered accordingly. In contrast, a white glow is visible in a well-developed lesion, suggesting a variation in treatment modality. Hence, dermoscopy assists in the selection of therapy in vitiligo.

Thus, three entities that manifest with hypopigmented or depigmented lesions on the face, especially in children, exhibit specific dermoscopic patterns. Therefore, dermoscopic assessment in these conditions is beneficial so that invasive diagnostic procedures such as biopsy can be avoided.

The limitations of the present study are the lack of histopathological examination, small sample size, and correlating clinical and dermoscopic findings irrespective of the duration of lesions.

\subsection{Conclusion}

Dermoscopy is a potential diagnostic tool differentiating various hypopigmentary and depigmentary disorders. It reduces the need for skin biopsy in cosmetic sensitive areas like the face and helps in the appropriate management of the condition.

\section{Footnotes}

Authors' Contribution: Study concept and design, B. A. and S.V.; Analysis and interpretation of data, B. A., E.E., and S.V.; Drafting of the manuscript, B.A. and S. V.; Critical revision of the manuscript for important intellectual content, B.A., E.E., and S. V.; Statistical analysis, M. R.

Conflict of Interests: The authors have no conflict of interest.

Data Reproducibility: The data presented in this study are openly available in one of the repositories or will be available on request from the corresponding author by this journal representative at any time during submission or after publication. Otherwise, all consequences of possible withdrawal or future retraction will be with the corresponding author.

\section{Ethical Approval: SNMC/IECHSR/2020-21/A-21a-1.1}

Funding/Support: The authors declare that this research received no funding/support.

Informed Consent: Written informed consent was taken from patients and concerned guardians.

\section{References}

1. Martin RF, Lugo-Somolinos A, Sanchez JL. Clinicopathologic study on pityriasis alba. BolAsocMed P R.1990;82(10):463-5. [PubMed: 2080961].

2. Karthikeyan K, Aishwarya M. Polymorphous Light Eruption- An Indian Scenario. Indian Dermatol Online J. 2021;12(2):211-9. doi: 10.4103/idoj.IDOJ_434_20. [PubMed: 33959517]. [PubMed Central: PMC8088173].

3. Gianfaldoni S, Tchernev G, Wollina U, Lotti J, Satolli F, Franca K, et al. Vitiligo in Children: A Better Understanding of the Disease. Open Access Maced J Med Sci. 2018;6(1):181-4. doi: 10.3889/oamjms.2018.040. [PubMed: 29484022]. [PubMed Central: PMC5816297].

4. Errichetti E, Ankad BS, Jha AK, Sonthalia S, Akay BN, Bakos R, et al. International Dermoscopy Society criteria for non-neoplastic dermatoses (general dermatology): Validation for skin of color through a Delphi expert consensus. Int J Dermatol. 2021. doi: 10.1111/ijd.15729. [PubMed: 34216025].

5. Malakar S, Mehta P. Ring Scales: A New Dermoscopic Sign in Polymorphous Light Eruption. Int J Dermatol. 2017;1(2):62-4. doi: 10.5005/jpjournals-10061-0015.

6. Errichetti E, Zalaudek I, Kittler H, Apalla Z, Argenziano G, Bakos $\mathrm{R}$, et al. Standardization of dermoscopic terminology and basic dermoscopic parameters to evaluate in general dermatology (nonneoplastic dermatoses): an expert consensus on behalf of the International Dermoscopy Society. Br J Dermatol. 2020;182(2):454-67. doi: 10.1111/bjd.18125. [PubMed: 31077336].

7. Lin RL, Janniger CK. Pityriasis alba. Cutis. 2005;76(1):21-4. [PubMed: 16144284].

8. Zaynoun ST, Aftimos BG, Tenekjian KK, Bahuth N, Kurban AK. Extensive pityriasis alba: A histological histochemical and ultrastructural study. Br J Dermatol. 1983;108(1):83-90. doi: 10.1111/j.13652133.1983.tb04582.x. [PubMed: 6821645].

9. Ankad BS, Koti VR. Dermoscopic approach to hypopigmentary or depigmentary lesions in skin of color. Clin Dermatol Rev. 2020;4(2):79.

10. Lembo S, Raimondo A. Polymorphic Light Eruption: What's New in Pathogenesis and Management. Front Med (Lausanne). 2018;5:252. 
doi: 10.3389/fmed.2018.00252.[PubMed:30250845].[PubMed Central: PMC6139322].

11. Kanwar AJ, Kumaran MS. Childhood vitiligo: Treatment paradigms. Indian J Dermatol. 2012;57(6):466-74. doi: 10.4103/0019-5154.103067. [PubMed: 23248365]. [PubMed Central: PMC3519254].

12. Thatte SS, Khopkar US. The utility of dermoscopy in the diagnosis of evolving lesions of vitiligo. Indian J Dermatol Venereol Leprol. 2014;80(6):505-8. doi: 10.4103/0378-6323.144144. [PubMed: 25382506].
13. Kumar Jha A, Sonthalia S, Lallas A, Chaudhary RKP. Dermoscopy in vitiligo: Diagnosis and beyond. Int J Dermatol. 2018;57(1):50-4. doi: 10.1111/ijd.13795. [PubMed: 29076154].

14. Vinay K, Ankad BS. Dermatoscopic Features of Pigmentary Diseases in Ethnic Skin. Indian Dermatol Online J. 2021;12(1):24-33. doi: 10.4103/idoj.IDOJ_561_20. [PubMed: 33768020]. [PubMed Central: PMC7982038]. 